\title{
Estimating Remainder Functionals by the Moduli of Smoothness .
}

\author{
P. Köhler
}

Abstract: For remainder functionals (e.g., approximation or quadrature errors), estimates by the moduli of smoothness are obtained. As a by-product, the constants in the estimate of the $\mathcal{K}$-functional by the moduli of smoothness are improved.

Keywords: Error estimates, modulus of continuity, modulus of smoothness, $\mathcal{K}$-functional AMS subject classification: $41 \mathrm{~A} 25,41 \mathrm{~A} 80$

\section{Introduction}

Let $R: C[a, b] \rightarrow \mathbb{R}$ be a bounded linear functional, and let $R\left[\mathcal{P}_{r-i}\right]=0$, where $\mathcal{P}_{r-1}$ are the polynomials of degree less $r$ (the setting in the following sections will be somewhat more general). The standard estimates for $R[f]$ are

$$
|R[f]| \leq\|R\|_{j}\left\|f^{(j)}\right\|_{\infty} \quad \text { for } f \in C^{j}[a, b] \text { and } 1 \leq j \leq r,
$$

where

$$
\|R\|_{j}=\sup \left\{\frac{|R[f]|}{\left\|f^{(j)}\right\|}: f \in C^{j}[a, b],\left\|f^{(j)}\right\|_{\infty} \neq 0\right\} .
$$

But if additional information on $f$ is available, e.g. $f^{(j)} \in$ Lip $\alpha$, this information cannot be used by estimates of the type (1.1). Therefore, it is of interest to have estimates by the moduli of continuity, i.e., estimates of the form

$$
|R[f]| \leq c(t) \omega_{r}(f, t)
$$

It is well known that such estimates exist, but, in general, no estimates for the constants $c(t)$ are available (see, e.g., Esser [3] and Ivanov [4]). The aim of this paper is to obtain

P. Köhler: Inst. Angew. Mathematik, TU Braunschweig, Pockelstr. 14, D - 38106 Braunschweig

ISSN 0232-2064 / \$2.50 C Heldermann Verlag Berlin 
$c(t)$ are available such estimates in the non-periodic case, and to improve them in the periodic case. Section 2 deals with functionals on $C_{2 \pi}^{s}$ and $L_{p, 2 \pi}^{s}$, Section 3 with functionals on $C^{s}[a, b]$, and Section 4 with the $\mathcal{K}$-functional.

\section{Functionals on $\mathbf{C}_{2 \pi}^{\mathrm{s}}$ and $\mathrm{L}_{\mathrm{p}, 2 \pi}^{\mathrm{g}}$}

Let $C_{2 \pi}^{s}$ be the class of $s$-times continuously differentiable, real-valued functions with period $2 \pi$, and $L_{p, 2 \pi}^{:}$the class of $2 \pi$-periodic, real-valued functions with absolutely continuous $(s-1)$-th and $p$-integrable $s$-th derivative, with

$$
\|f\|_{p}^{p}=\int_{0}^{2 \pi}|f(x)|^{p} d x \quad(1 \leq p<\infty) \quad \text { and } \quad\|f\|_{\infty}=\sup _{0 \leq x \leq 2 \pi}|f(x)| .
$$

For convenience, we denote these classes by $X_{p}^{s}$, i.e., $X_{\infty}^{s}=C_{2 \pi}^{s}$ and $X_{p}^{s}=L_{p, 2 \pi}^{s}$ for $1 \leq p<\infty$. We.consider functionals

$$
R: X_{p}^{s} \rightarrow \mathbb{R}
$$

satisfying

$$
|R[f+g]| \leq|R[f]|+|R[g]|
$$

and

$$
\|R\|_{i, p}=\sup \left\{\frac{|R| f] \mid}{\left\|f^{(i)}\right\|_{p}}: f \in X_{p}^{i},\left\|f^{(i)}\right\|_{p} \neq 0\right\}<\infty
$$

for $i=s, \ldots, r$, for some $r>s \geq 0$. We therefore have

$$
|R[f]| \leq\|R\|_{i, p}\left\|f^{(i)}\right\|_{p} \quad \text { for } f \in X_{p}^{i}, \quad i=s, \ldots, r .
$$

Further let

$$
\omega_{j}(f, t)_{p}=\sup _{|h| \leq t}\left\|\Delta_{h}^{j} f\right\|_{p}, \quad \text { where } \quad \Delta_{h}^{j} f(x)=\sum_{i=0}^{j}\left(\begin{array}{l}
j \\
i
\end{array}\right)(-1)^{j-i} f(x+i h),
$$

be the $j$-th modulus of smoothness (for the properties of $\omega_{j}$, see Schumaker [12: Chap. 2.8]). To simplify notation, we introduce the following abbreviations. Let

$$
\lceil x\rceil=\min \{i \in \mathbb{Z}: i \geq x\}, \quad \beta_{r}=\left(\begin{array}{c}
r \\
\lceil r / 2\rceil
\end{array}\right)
$$

and

$$
\rho_{r}=\int_{0}^{r / 2}\lceil x\rceil^{r} N_{r}\left(x+\frac{r}{2}\right) d x=\sum_{i=1}^{\left\lceil\frac{r}{2}\right\rceil} i^{r} N_{r+1}\left(i+\frac{r}{2}\right) \leq \frac{1}{2}\left\lceil\frac{r}{2}\right\rceil^{r}
$$

$\left(\rho_{1}=1 / 2, \rho_{2}=1 / 2, \rho_{3}=31 / 48, \rho_{4}=9 / 8\right)$. Here, $N_{r}$ is the B-spline of degree $r-1$ for the knots $0,1, \ldots, r$, satisfying

$$
\int_{0}^{r} N_{r}(x) d x=1
$$


Further, there holds

$$
\int_{t-1}^{t} N_{r}(x) d x=N_{r+1}(t)
$$

(Schoenberg [11: p. 12]; this has been used to obtain the second formula for $\rho_{r}$ given in $(2.4))$, and

$$
\int_{0}^{r} F^{(r)}(x+\alpha v) N_{r}(v) d v=\alpha^{-r} \Delta_{\alpha}^{r} F(x) \quad \text { for } F \in X_{1}^{r}
$$

and $\alpha>0$ (Schumaker [12: p. 54]). The following lemma is well known (see, e.g., DeVore [2]), but we have modified the proof such that better constants are obtained.

Lemma 2.1. Let $r \geq 1$ and $1 \leq p \leq \infty$. Then for all $f \in X_{p}^{0}$ and all $t>0$, there exists a function $g \in X_{p}^{r}$ such that or

a) $\|f-g\|_{p} \leq 2 \beta_{r}^{-1} \rho_{r} \omega_{r}(f, t)_{p}$ and $\left\|g^{(r)}\right\|_{p} \leq \beta_{r}^{-1}\left(2^{r}-\beta_{r}\right) t^{-r} \omega_{r}(f, t)_{p}$, .

b) $\|f-g\|_{p} \leq \beta_{r}^{-1} \omega_{r}(f, t)_{p}$ and $\left\|g^{(r)}\right\|_{p} \leq\left\lceil\frac{r}{2}\right\rceil^{r} \delta_{r} t^{-r} \omega_{r}(f, t)_{p}$, where $\delta_{1}=\delta_{2}=1$ and $\delta_{r}=2$ for $r \geq 3$.

Proof. Let $\alpha_{r}=\lceil r / 2\rceil$, and let $g$ be defined by

$$
\begin{aligned}
g(x) & =\beta_{r}^{-1} \int_{-r / 2}^{r / 2}\left(\beta_{r} f(x)+(-1)^{r-\alpha_{r}-1} \Delta_{v t}^{r} f\left(x-\alpha_{r} v t\right)\right) N_{r}\left(\dot{v}+\frac{r}{2}\right) d v \\
& =\beta_{r}^{-1} \sum_{\substack{i=0 \\
i \neq \alpha_{r}}}^{r}\left(\begin{array}{l}
r \\
i
\end{array}\right)(-1)^{i-\alpha_{r}-1}\left(\left(i-\alpha_{r}\right) t\right)^{-r} \Delta_{\left(i-\alpha_{r}\right) i}^{r} F\left(x+\frac{r}{2}\left(\alpha_{r}-i\right) t\right)
\end{aligned}
$$

by $(2.7)$, where $F^{(r)}=f$.

a) Minkowski's inequality and the periodicity of $f$ yield

$$
\begin{aligned}
\|f-g\|_{p} & \leq \beta_{r}^{-1} \int_{-r / 2}^{+r / 2}\left\|\Delta_{v t}^{r} f\right\|_{p} N_{r}\left(v+\frac{r}{2}\right) d v \\
& \leq \beta_{r}^{-1} \int_{-r / 2}^{+r / 2} \omega_{r}(f,|v t|)_{p} N_{r}\left(v+\frac{r}{2}\right) d v \\
& \leq \beta_{r}^{-1} \int_{-r / 2}+r / 2\lceil\|v\|\rceil^{r} \omega_{r}(f, t)_{p} N_{r}\left(v+\frac{r}{2}\right) d v \\
& =2 \beta_{r}^{-1} \rho_{r} \omega_{r}(f, t)_{p},
\end{aligned}
$$

while, by the triangle inequality,

$$
\left\|g^{(r)}\right\|_{p} \leq \beta_{r}^{-1} \sum_{\substack{i=0 \\
i \neq \alpha_{r}}}^{r}\left(\begin{array}{l}
r \\
i
\end{array}\right)\left|i-\alpha_{r}\right|^{-r} t^{-r}\left\|\Delta_{\left(i-\alpha_{r}\right) s}^{r} F^{(r)}\right\|_{p}
$$




$$
\begin{aligned}
& \leq \beta_{r}^{-1} t^{-r} \sum_{\substack{i=0 \\
i \neq \alpha_{r}}}^{r}\left(\begin{array}{l}
r \\
i
\end{array}\right)\left|i-\alpha_{r}\right|^{-r} \omega_{r}\left(f,\left|i-\alpha_{r}\right| t\right)_{p} \\
& \leq \beta_{r}^{-1} t^{-r} \sum_{\substack{i=0 \\
i \neq \alpha_{r}}}^{r}\left(\begin{array}{l}
r \\
i
\end{array}\right) \omega_{r}(f, t)_{p} \\
& =\beta_{r}^{-1} t^{-r}\left(2^{r}-\beta_{r}\right) \omega_{r}(f, t)_{p} .
\end{aligned}
$$

b) From (2.9), we also get

$$
\left\|g^{(r)}\right\|_{p} \leq \beta_{r}^{-1} t^{-r} \sum_{\substack{i=0 \\
i \neq \alpha_{r}}}^{r}\left(\begin{array}{l}
r \\
i
\end{array}\right)\left|i-\alpha_{r}\right|^{-r} \omega_{r}\left(f, \alpha_{r} t\right)_{p} \leq \delta_{r} t^{-r} \omega_{r}\left(f, \alpha_{r} t\right)_{p}
$$

since $\beta_{r}^{-1} \sum_{\substack{i=0 \\ i \neq \alpha_{r}}}^{r}\left(\begin{array}{l}r \\ i\end{array}\right)\left|i-\alpha_{r}\right|^{-r} \leq \delta_{r}$, by some elementary calculations, while (2.8) together with (2.5) gives

$$
\|f-g\|_{p} \leq \beta_{r}^{-1} \omega_{r}\left(f, \frac{r}{2} t\right)_{p} \leq \beta_{r}^{-1} \omega_{r}\left(f, \alpha_{r} t\right)_{p}
$$

Replacing $u=\alpha_{r} t$ proves part b)

From Lemma 2.1, we get the following estimates for $|R[f]|$ in terms of the moduli of smoothness.

Theorem 2.1. Let $R: X_{p}^{s} \rightarrow \mathbb{R}$ be a functional satisfying (2.1) and (2.3), and let $t>0$ and $1 \leq j \leq r-s$. Then

$$
|R[f]| \leq \beta_{j}^{-1} \cdot\left(2 p_{j}\|R\|_{s, p}+\left(2^{j}-\beta_{j}\right) t^{-j}\|R\|_{j+s, p}\right) \omega_{j}\left(f^{(o)}, t\right)_{p} \quad \text { for } f \in X_{p}^{s} .
$$

Proof. Let $F=f^{(\bullet)} \in X_{p}^{0}$. By Lemma 2.1, there exists $G \in X_{p}^{j}$ such that

$$
\|F-G\|_{p} \leq c_{1} \omega_{j}(F, t)_{p} \quad \text { and } \quad\left\|G^{(j)}\right\|_{p} \leq c_{2} \omega_{j}(F, t)_{p} .
$$

Now choose $g \in X_{p}^{j+s}$ with $g^{(\bullet)}=G$. Then

$$
\begin{aligned}
|R[f]| & \leq|R[f-g]|+|R[g]| \\
& \leq\|R\|_{s, p}\left\|f^{(0)}-g^{(o)}\right\|_{p}+\|R\|_{j+s, p}\left\|g^{(j+o)}\right\|_{p} \\
& =\|R\|_{\infty, p}\|F-G\|_{p}+\|R\|_{j+o, p}\left\|G^{(j)}\right\|_{p} .
\end{aligned}
$$

Inserting the estimates from (2.10), together with the constants of Lemma 2.1a) (with $r$ replaced by $j$ ) completes the proof

Example 2.1. Let $E_{n}[f]=\inf _{g \in T_{n}}\|f-\grave{g}\|_{\infty}$ be the error in the approximation of $f \in C_{2 \pi}$ by trigonometric polynomials of degree lesser or equal $n$. By the Theorem of Favard-Achieser-Krein,

$$
\left\|E_{n}\right\|_{r, \infty}=K_{r}(n+1)^{-r} \quad \text { where } K_{r}=\frac{4}{\pi} \sum_{\nu=0}^{\infty}\left(\frac{(-1)^{\nu}}{2 \nu+1}\right)^{r+1} \leq \frac{\pi}{2}
$$


is Favard's constant. Choosing $t=2 /(n+1)$ in Theorem 2.1 , we obtain

$$
E_{n}[f] \leq \frac{\pi c_{j}}{(n+1)^{s}} \omega_{j}\left(f^{(s)}, \frac{2}{n+1}\right)_{\infty} \quad \text { for } f \in C_{2 \pi}^{s},
$$

$s \geq 0$ and $j \geq 1 ;$ where

$$
c_{j}=\frac{1}{\beta_{j}}\left(\rho_{j}+\frac{1}{2}\right)-\frac{1}{2^{j+1}}
$$

$\left(c_{1}=3 / 4, c_{2}=3 / 8, c_{3}=23 / 72\right.$ and $c_{4}=23 / 96$, but $c_{j} \rightarrow \infty$ for $\left.j \rightarrow \infty\right)$. The asymptotic behaviour of $c_{r}$ is probably be given by

$$
c_{r} \sim \frac{\sqrt{\pi r}}{2}\left(\frac{r}{4 \sqrt{3 e}}\right)^{r} \quad \text { for } r \rightarrow \infty
$$

but we have not been able to prove this.

It can be shown that Theorem 2.1 remains true if $\|R\|_{i, p}$ is replaced by

$$
\|R\|_{i, p}=\sup \left\{\frac{|R[f]|}{\left\|f^{(i)}\right\|_{p}}: f \in X_{p}^{i},\left\|f^{(i)}\right\|_{p} \neq 0, \int_{0}^{2 \pi} f^{(i)}(x) d x=0\right\}
$$

(obviously, $\|R\|_{i, p} \leq\|R\|_{i, p}$, but with equality for $i \geq 1$ because of the periodicity of the functions considered). E.g., for quadrature errors

$$
R[f]=\int_{a}^{b} f(x) d x-\sum_{i=1}^{n} a_{i} f\left(x_{i}\right)
$$

there holds

$$
\|R\|_{0, \infty}=b-a+\sum i=1^{n}\left|a_{i}\right|, \text { but }\|R\|_{0, \infty}=\sum_{i=1}^{n}\left|a_{i}\right|
$$

(for an example and some other details omitted here, see [6]).

\section{Functionals on $\mathbf{C}^{\mathbf{s}}[\mathbf{a}, \mathbf{b}]$}

Let $C^{\cdot}[a, b]$ be the class of $s$-times continuously differentiable, real-valued functions on $[a, b]$, and $\|f\|=\sup |f(x)|$. We consider functionals $R: C^{\prime}[a, b] \rightarrow \mathbb{R}$ satisfying (2.1), and assume that, for $f \in C^{i}[a, b]$ and $i=s, \ldots, r$,

$$
|R[f]| \leq\|R\|_{i}\left\|f^{(i)}\right\|
$$

holds, where $\|R\|_{i}$ is defined analogous to (2.2), and $\|R\|_{i}<\infty$. Further, let

$$
\omega_{j}(f, t)=\sup \left\{\left|\Delta_{h}^{j} f(x)\right|:|h| \leq t \text { and } x, x+j h \in[a, b]\right\}
$$

denote the $j$-th modulus of continuity of $f$. The proof of an analogue of Lemma 2.1 is more complicated, since $f$ is not defined outside $[a, b]$. A standard method to overcome this difficulty is to extend $f$ in a suitable way (see DeVore [2]), but then one has to know the constants related to this extension. For the case of the sup-norm considered in this 
section, this difficulties can be avoided by a modification of the step size of the differences involved in the proof (this does not work for the $L_{p}$-norms, $1 \leq p<\infty$, which is the reason why we do not treat this case here). This modification is a useful tool to obtain explicit constants, when Steklov functions are applied to non-periodic functions. It was derived by the author some years ago for a first version of this paper, but afterwards, I discovered that it had already been used by Sendov in [9]; inserting $t=(b-a) / r^{2}$ in part b) of the following lemma, gives the estimate of Sendov. Let

$$
\tau_{r}=\int_{0}^{r}\lceil x\rceil^{r} N_{r}(x) d x=\sum_{i=1}^{r} i^{r} N_{r+1}(i) \leq r^{r} .
$$

$\left(\tau_{1}=1, \tau_{2}=5 / 2, \tau_{3}=10, \tau_{4}=331 / 6\right.$; the second formula for $\tau$ follows from (2.6)).

Lemma 3.1. Let $f \in C[a, b], r \geq 1$ and $t \in\left(0,(b-a) / r^{2}\right]$. Then there exists $a$ function $g \in C^{r}[a, b]$ such that

a) $\|f-g\| \leq \tau_{r} \omega_{r}(f, t)$ and $\left\|g^{(r)}\right\| \leq\left(2^{r}-1\right) t^{-r} \omega_{r}(f, t)$,

or

b) $\|f-g\| \leq \omega_{r}(f, r t)$ and $\left\|g^{(r)}\right\| \leq(r+1) t^{-r} \omega_{r}(f, r t)$.

Proof. Let

$$
g(x)=\int_{0}^{r}\left(f(x)+(-1)^{r+1} \Delta_{u}^{r} f(x)\right) N_{r}(v) d v \quad \text { with } \quad u=v t-\frac{x-a}{b-a} r t .
$$

Here, $u$ and the restriction for $t$ stated in the lemma have been chosen such that $x+i u \in$ $[a, b]$ always (i.e., for $x \in[a, b]$ and $i=0, \ldots, r$ ).

a) We obtain

$$
|f(x)-g(x)| \leq \int_{0}^{r} \omega_{r}\left(f, t\left|v-\frac{x-a}{b-a} r\right|\right) N_{r}(v) d v .
$$

For simplicity, let $a=0$ and $b=1$, and let

$$
\phi(x)=\omega_{r}(f, t x) \quad \text { and } \quad \psi(x)=\int_{0}^{r} \phi(|v-r x|) N_{r}(v) d v
$$

From the monotonicity of $\dot{\omega}_{r}(f, \cdot)$, it follows that $\dot{\phi}$ is an increasing function, and the symmetry of $N_{r}$ yields $\psi(1-x)=\psi(x)$. Using the symmetry properties of $N_{r}$ and $\psi$, and the monotonicity of $\phi$ and of $N_{r}$ on $[0, r / 2]$, we obtain for $x \in[0,1 / 2]$

$$
\begin{aligned}
\psi(0)-\psi(x) & =\int_{0}^{r x}(\phi(v)-\phi(r x-v)) N_{r}(v) d v+\int_{r x}^{r}(\phi(v)-\phi(v-r x)) N_{r}(v) d v \\
\geq & \int_{0}^{r x}(\phi(v)-\phi(r x-v)) N_{r}(v) d v \\
& =\int_{0}^{r x / 2}(\phi(r x-v)-\phi(v))\left(N_{r}(r x-v)-N_{r}(v)\right) d v \\
\geq & 0
\end{aligned}
$$


Because of the symmetry, the same holds for $x \in[1 / 2,1]$, so that

$$
\|f-g\| \leq \psi(0)=\int_{0}^{r} \omega_{r}(f, t v) N_{r}(v) d v \leq \int_{0}^{r}\lceil v\rceil^{r} N_{r}(v) d v \omega_{r}(f, t)=\tau_{r} \omega_{r}(f, t) .
$$

For the $r$-th derivative of $g$, we obtain, using (2.7),

$$
\begin{aligned}
\left|g^{(r)}(x)\right|^{\top} & =\left|\sum_{i=1}^{r}\left(\begin{array}{l}
r \\
i
\end{array}\right)(-1)^{1-i}(i t)^{-r}\left(1-\frac{i r t}{b-a}\right)^{r} \Delta_{i t}^{r} f\left(x-\frac{x-a}{b-a} i r t\right)\right| \\
& \leq \sum_{i=1}^{r}\left(\begin{array}{l}
r \\
i
\end{array}\right)(i t)^{-r} \omega_{r}(f, i t) \\
& \leq \sum_{i=1}^{r}\left(\begin{array}{l}
r \\
i
\end{array}\right) t^{-r} \omega_{r}(f, t) \\
& =\left(2^{r}-1\right) t^{-r} \omega_{r}(f, t) .
\end{aligned}
$$

b) From (3.4), we also get

$$
\left|g^{(r)}(x)\right| \leq \sum_{i=1}^{r}\left(\begin{array}{l}
r \\
i
\end{array}\right) i^{-r} t^{-r} \omega_{r}(f, r t) \leq(r+1) t^{-r} \omega_{r}(f, r t) .
$$

Further, $\|f-g\| \leq \omega_{r}(f, r t)$, by (3.3) and (2.5)

In the same way as Theorem 2.1, we obtain the following one.

Theorem 3.1. Let $R: C^{s}[a, b] \rightarrow \mathbb{R}$ be a functional satisfying (2.1) and (3.1), and let $1 \leq j \leq r-s$ and $t \in\left(0,(b-a) / j^{2}\right]$. Then

$$
|R[f]| \leq\left(\tau_{j}\|R\|_{a}+\left(2^{j}-1\right) t^{-j}\|R\|_{j+a}\right) \omega_{j}\left(f^{(s)}, \dot{t}\right) \quad \text { for } f \in C^{s}[a, b]
$$

The estimate of Theorem 3.1 makes use of $\|R\|_{s}$ and $\|R\|_{j+s}$, which, however, may not be known. This difficulty can be overcome if $\|R\|_{m}$ and $\|R\|_{r}$ are known for some $m<s$ and some $r>j+s$, by using the estimate

$$
\|R\|_{i} \leq \dot{K}_{i-m}\|R\|_{m}^{(r-i) /(r-m)}\left(\frac{\|R\|_{r}}{K_{r-m}}\right)^{(i-m) /(r-m)}
$$

which holds for $0 \leq m \leq i \leq r$ (see Ligun [7] and Köhler [5]; the $K_{i}$ are again Favard's constants):

Example 3:1. Let $R_{n}^{G}[f]=\int_{-1}^{1} f(x) d x-Q_{n}^{G}[f]$ be the error of the $n$-point GaussLegendre quadrature formula. It is well known that

$$
\left\|R_{n}^{G}\right\|_{0}=4 \quad \text { and } \quad\left\|R_{n}^{G}\right\|_{2 n}=\frac{2^{2 n+1} n !^{4}}{(2 n+1)(2 n) !^{3}}
$$


Using Stirling's and Wallis' formula, it can be shown that

$$
t_{2 n}:=\left(\frac{\left\|R_{n}^{G}\right\|_{2 n}}{\left\|R_{n}^{G}\right\|_{0} K_{2 n}}\right)^{1 /(2 n)} \leq \frac{e}{4 n} .
$$

Using (3.5) to estimate $\left\|R_{n}^{G}\right\|_{s}$ and $\left\|R_{n}^{G}\right\|_{j+s}$ by $\left\|R_{n}^{G}\right\|_{0}$ and $\left\|R_{n}^{G}\right\|_{2 n}$, and applying Theorem 3.1 with $r=2 n$ and $t=t_{2 n}$, yields

$$
\left|R_{n}^{G}[f]\right| \leq 4\left(\frac{e}{4 n}\right)^{s}\left(\tau_{j} K_{s}+\left(2^{j}-1\right) K_{j+s}\right) \omega_{j}\left(f^{(s)}, \frac{e}{4 n}\right) \quad \text { for } f \in C^{s}[a, b]
$$

$0 \leq s<j+s \leq 2 n$ and $n \geq e j^{2} / 8$.

Finally, let us shortly consider compound functionals, and state an estimate given by Sendov and Popov [10: p. 49] for the $\tau$-moduli, in the framework of this paper. Let the function $R: C^{\bullet}[0,1] \rightarrow \mathbb{R}$ satisfy $(2.1)$ and (3.1), and let the $N$-compound functional $R_{N}: C^{\bullet}[a, b] \rightarrow \mathbb{R}$ be defined by

$$
R_{N}[f]=\frac{b-a}{N} \sum_{i=0}^{N-1} R\left[f\left(a+(i+\cdot) \frac{b-a}{N}\right)\right] \quad \text { for } f \in C^{s}[a, b] .
$$

Theorem 3.2. Let $1 \leq j \leq r-s$. Then

$$
\mid R_{N}[f]\left\|\leq 6 \frac{(b-a)^{a+1}}{N^{\circ}}\right\| R \|, \omega_{j}\left(f^{(s)}, \frac{b-a}{N(j+1)}\right) \quad \text { for } f \in C^{s}[a, b]
$$

Especially, this can be applied to compound quadrature rules. Better (and partly sharp) estimates by $\omega_{r}(f, \cdot)$ have been obtained by Büttgenbach, Lüttgens and Nessel [1] for the compound midpoint and the first four compound Newton-Cotes rules, using representations for the error by differences of order $r$.

\section{Estimates for the $\mathcal{K}$-functional}

a) Let us first consider the $\mathcal{K}$-functional of Peetre in the periodic case, i.e.,

$$
\mathcal{K}_{r}(f, u)_{p}=\inf _{g \in X_{p}^{r}}\left(\|f-g\|_{p}+u\left\|g^{(r)}\right\|_{p}\right) \quad \text { for } f \in X_{p}^{0} .
$$

Specializing to $g$ from Lemma 2.1a), and choosing $t=2 u$, yields the following theorem.

Theorem 4.1. Let $1 \leq p \leq \infty, r \geq 1$ and $u>0$. Then

$$
\mathcal{K}_{r}\left(f, u^{r}\right)_{p} \leq 2 c_{r} \omega_{r}(f, 2 u)_{p} \quad \text { for } f \in X_{p}^{0}
$$

with $c_{r}$ as in (2.11). 
For $r=1,2$, this coincides with the estimate given by Maligranda [8]. The unmodified proof of Lemma 2.1, as given by DeVore [2], yields $\mathcal{K}_{r}\left(f, u^{r}\right)_{p} \leq\left(r^{r}+1-2^{-r}\right) \omega_{r}(f, 2 u)_{p}$ (see also Maligranda [8]).

b) For the $\mathcal{K}$-functional in the non-periodic case,

$$
\mathcal{K}_{r}(f, u)=\inf _{g \in C^{r}[a, b]}\left(\|f-g\|+u\left\|g^{(r)}\right\|\right) \quad \text { for } f \in C[a, b],
$$

choose, e.g., $g$ from Lemma 3.1a) with $t=2 u$.

Theorem 4.2. Let $r \geq 1$ and $0<u \leq(b-a) /\left(2 r^{2}\right)$. Then

$$
\mathcal{K}_{r}\left(f, u^{r}\right) \leq\left(\tau_{r}+1-2^{-r}\right) \omega_{r}(f, 2 u) \quad \text { for } f \in C[a, b] .
$$

It is well known that $\mathcal{K}_{r}$ can be estimated by $\omega_{r}$, but, as far as we know, no explicit constants have been given for $r>2$.

\section{References}

[1] Büttgenbach, B., Lüttgens, G. and R.J. Nessel: An explicit representation'of the remainder of some Newton-Cotes formulas in terms of higher order differences. Z. Anal. Anw. 11 (1992), 135 - 141.

[2] DEVORE, R.A.: Degree of approximation. In: Approximation Theory II (eds.: G.G. Lorentz, C.K. Chui and L.L. Schumaker). New York: Academic Press 1976, pp. $117-161$.

[3] EsSER, H.: Abschätzungen durch Stetigkeitsmoduli bei Folgen von linearen Funktionalen. Lect. Notes Math. 556 (1976), $184-190$.

[4] IVANOV, K.G.: New estimates of errors of quadrature formulae, formulae of numerical differentiation and interpolation. Anal. Math. 6 (1980), $281-303$.

[5] KöHLER, P.: Intermediate error estimates. J. Comp. Appl. Math. (to appear).

[6] KöHLER, P.: Estimating remainder functionals by the moduli of smoothness. Report. Braunschweig: Institute für Mathematik, Bericht 93/15.

[7] Ligun, A.A.: Exact inequalities for the upper bounds of seminorms on a class of periodic functions. Math. Notes 13 (1973), $389-393$.

[8] Maligranda, L.: Interpolation spaces in the theory of approximation. In: Methods of Functional Analysis in Approximation Theory (ISNM 76) (eds.: C.A. Micchelli, D.V. Pai and B.V. Limaye). Basel: Birkhäuser Verlag 1986, pp. $264-279$.

[9] Sendov, Bl.: A modified Steklov function (in Russian). Comp. Rend. Acad. Bulg. Sci. 36 (1983), $315-317$.

[10] Sendov, BL. and V.A. Popov: The Averaged Moduli of Smoothness. Chichester: Wiley 1988.

[11] Schoenberg, I.J.: Cardinal Spline Interpolation. Philadelphia: SIAM 1973.

[12] SchUmakeR, L.L.: Spline Functions. New York: Wiley 1981.

Received 12.07.1993, in revised form 04.10 .1983 